\title{
El desafío del desarrollo local: una agenda para la discusión en América Latina
}

\author{
Isaías Lescher Soto*
}

\section{Resumen}

Las tendencias mundiales en el debate sobre el desarrollo han incluido en sus temáticas la dimensión de lo local como parte de este complejo proceso de transforma. ción societal. El presente trabajo tiene como fin realizar una reflexión sobre el desarrollo local, haciendo una revisión de su conceptualización, sus componentes, las relaciones entre estos, así como las amenazas y oportunidades de esta propuesta en América Latina. Se concluye que el desarrollo local es un proceso multidimensional y que su viabilidad depende del surgimiento de iniciativas locales autónomas, de una descentralización efectiva y de la participación de diversos actores en la formulación de un proyecto político que establezca como meta la búsqueda de bienestar, la democracia y la equidad para todos los ciudadanos de la comunidad local, en armonía con su ambiente y con los intereses de la comunidad nacional a la que pertenecen.

Palabras clave: Desarrollo local, comunidad local, actores-agentes, descentralización, proyecto político.

\section{Local Development Challenge a Discussion Agenda in Latin America}

\section{Abstract}

In the debate world tendencies about development have included the local development dimension as a part of this transformation complex societal process in their topics. The purpose of this work is to reflect upon the local development, making a review its con-

Recibido: 16-09-97 . Aceptado: 28-10-97

- Sociólogo, Docente de la Escuela de Sociología de LUZ, participante de la Maestría en Desarrollo Social de LUZ. Dirección: Calle 60B, No. 9B-73. Maracaibo-Venezuela 
ceptualization, its components, the relationship between them, as well as its threats and opportunities of this proposition in Latin America. It is concluded that local development is a multidimensional process and its feasibility depends on independent local iniciatives spouting, an effective decentralization and all actors participation in formulating a political project which establishes the research of welfare, democracy, and the equity for all local community people in harmony with its environment and the national community interests they belong to.

Key words: Local Development, Local Community, Actors-Agents, Political Project, Descentralization.

\section{Antecedentes}

La génesis de la discusión sobre el desarrollo local se enmarca en la crisis económico- social generada en los países in. dustrializados a finales de la década de los años setenta. El fin del crecimiento económico sostenido, la aparición de estrategias de desarrollo de corte monetarista, así como la emergencia del desempleo, introdujeron dudas acerca de la posibilidad de mantener los estándares de vida hasta ese momento logrados.

Al mismo tiempo, la coexistencia de regiones hiperindustrializadas con otras en procesos de regresión; y el deterioro de las tradicionales áreas de crecimiento industrial como el siderúrgico (Arocena, 1995: 30), coadyuvó a esta situación de cuestionamiento del desarrollo logrado, el cual estaba íntimamente relacionado con la concentración en algunas zonas geográficas de grandes aparatos industriales.

Comienza así a cobrar importancia la necesidad de aprovechar iniciativas de desarrollo de menor escala ligadas a dinámicas locales con una fuerte movilización del potencial humano, económico y hasta geográfico.
Estas iniciativas han estado relacionadas en los países industrializados con el reconocimiento de la existencia de tradiciones, normas y valores que conforman identidades arraigadas (Cárdenas, 1996: 2), que han impulsado acciones en respuesta a la crisis mencionada.

También se ha hablado de ciertas "megatendencias universales" (Boisier, 1992: 116) que tienden a vitalizar proyectos políticos locales ligados a procesos de descentralización.

Entre estas condiciones se pueden mencionar:

a) La revolución científico - tecnológica que ha implicado la emergencia de una "economía de geometría variable" caracterizada por la instrumentación de modelos postfordistas en la organización del trabajo industrial, la reducción del poder del sindicalismo, (lo cual ha implicado una nueva relación capital trabajo), la preeminencia de la tecnología de producción basada en insumos de conocimiento y finalmente la incorporación de procesos de desconcentración o descentralización del asentamiento industrial. Este esquema de economía de "geometría variable" está relacionado con la posibilidad de una 
mayor descentralización del poder de decisión a nivel territorial.

b) El proceso de reestructuración política y administrativa del Estado. Esta condición está íntimamente ligada a lo anterior, puesto que la transformación del sistema de producción industrial, ha puesto en entredicho la función de acumulación del Estado. Se plantea una discusión en la que se establece que ni las formas tradicionales de acumulación directa del Estado a través de la propiedad pública, ni las formas indirectas de apoyo a través de la política pública, son ya útiles, por lo que el Estado deberia insertarse en funciones como las de apoyo a la investigación científica y tecnológica y políticas de inserción internacional en vez de políticas de fomento industrial.

c) Creciente y universal demanda de cuerpos organizados de la sociedad civil; muchos de estos relacionados con procesos territoriales en la búsqueda de mayores espacios de autorrealización, lo que supone descentralización y autonomía de acción. En esta tendencia están involucradas las necesidades de autogobierno y autorrepresentación, la desconfianza en organizaciones nacionales y centralizadas, asi como también factores de tipo étnico, religioso, económico y político. Es decir, movimientos sociales que se caracterizan por ser simultáneamente un conflicto social y un proyecto cultural, aspirando a la realización de valores culturales (Touraine, 1992: 238). Estos movimientos cuestionan la dominación ejercida por un Estado central, bus- cando mayores cuotas de participación.

d) Tendencia a la privatización de actividades y de servicios, traspasando funciones en la producción y distribución de recursos a entes dotados de personalidad jurídica distinta al Estado.

En este orden de ideas, lo que se pone de manifiesto es un nuevo pacto social entre el Estado y la sociedad civil (Boisier, 1992: 11) en el que esta pueda asumir nuevas responsabilidades en los diferentes espacios en los que antes el Estado era el principal agente.

En general esas "megatendencias universales" han contribuido a revalorizar la potencialidad del desarrollo local como instrumento y fin del desarrollo global de las sociedades.

En otra perspectiva, a nivel Latinoamericano, la discusión sobre lo local ha estado condicionada por la crisis de la estrategia de desarrollo en la que el Estado era el motor de las transformaciones económicas y sociales, aunada a las penurias de legitimidad y representatividad de los actores políticos ligados a estos procesos. Finalmente la creciente necesidad de reencontrarse con la identidad cultural que se vive hoy en las sociedades de esta parte del mundo, como consecuencia de las dinámicas democratizadoras que se han insertado como formas de organización política.

En lo subsiguiente, este artículo abordará el desafío del desarrollo local como una alternativa estratégica a ser discutida en América Latina, proponiendo una agenda para su discusión que incluye un nivel teórico - conceptual sobre este proceso y un nivel analítico que iden- 
tifica las principales amenazas y oportunidades que influyen en la viabilidad del mismo en dicha región.

\section{Conceptualización del desarrollo local}

El desarrollo local puede ser conceptualizado como un proceso dinámico y sistémico a partir del cual, dentro de uno terrltorio (parte de una comunidad nacional) un conjunto de actores - agentes de desarrollo local construyen e instrumentan un proyecto político, que garantiza el progreso de la comunidad local a través de la creación de espacios políticos representativos de participación, la construcción de la ciudadanía y de la identidad local, la satisfacción de las necesidades básicas de la población, la equidad en la distribución de los recursos y el crecimiento productivo de esa localidad, garantizando el mantenimiento del medio ambiente.

Esta conceptualización involucra que el desarrollo local esté compuesto de elementos tales como: territorio, comunidad local, actores - agentes del desarrollo local, proyecto político y comunidad nacional.

a) El Territorio: Constituye el espacio físico delimitado donde se generarán las actividades de los individuos pertenecientes a una comunidad local.

"Estos espacios se vuelven significativos para el grupo que los habita, se cargan de sentido porque por él transitaron generaciones que fueron dejando huellas, las trazas de su trabajo, los efectos de su acción de transformación de la naturaleza. En esos territorios emergen inequivocas señales de destrucción y construcción propias de la especie humana. Son espacios penetrados por las formas de vida de los hombres que los habitan, por sus ritos, sus costumbres, sus valores, sus creencias". (Arocena, 1995: 24). En este sentido, en la conciencia del individuo se genera una identidad con este territorio y con el otro que lo habita, su historia, su presente y su futuro.

b) Comunidad Local: puede ser definida como el conjunto de individuos, grupos sociales y actores sociales que se interrelacionan entre sí en la dimensión económica generadora de riqueza, compartiendo a su vez una historia, una identidad colectiva común expresada en valores y normas interiorizadas por sus miembros, que están interconectadas en un sistema de relaciones de poder constituido en torno a la posesión de recursos económicos, políticos, sociales y culturales. Esta comunidad local forma parte de una sociedad global llamada comunidad nacional, con la cual comparte análogas relaciones pero en una dimensión social integradora de las especificidades regionales.

c) Actores - Agentes del Desarrollo Local: Están constituidos por aquellos individuos, grupos o instituciones capaces de elaborar e impulsar la ejecución de propuestas políticas para la conducción de la comunidad local, estableciendo lineamientos de acción políticos, económicos, sociales y culturales con el fin de aprovechar las fortalezas y oportunidades de la región y disminuir las amenazas y debilidades de la misma. Coincidiendo con Arocena (1995: 26), no todo individuo, grupo o institu- 
ción será considerado como actor agente del desarrollo local, si no son portadores de propuestas que tiendan a capitalizar las potencialidades locales.

d) Proyecto Político Local: Es la articulación de las diversas iniciativas generadas por los actores - agentes del desarrollo local, traducida en una serie de objetivos y metas en las dimensiones política, social, económica y cultural; las estrategias y tácticas para lograr tales objetivos y la identificación de agentes responsables de cada acción a realizar.

e) Comunidad Nacional: No puede hablarse de desarrollo local, si no se le contextualiza en el marco de un desarrollo social y nacional. Bajo esta perspectiva el desarrollo de las comunidades regionales será el fin, el medio y la estrategia para el logro del progreso conjunto de individuos, grupos sociales y actores que comparten un territorio, una historia y una identidad nacionales. El desarrollo local debe formar parte de una estrategia de desarrollo nacional en el que considerando las diversidades regionales, se cree un proceso sinérgico y aglutinador de las potencialidades, inscribiendo iniciativas locales en procesos sistémicos de carácter nacional.

\section{El desarrollo local como proceso}

El desafío del desarrollo local, es un reto que cada sociedad debe enfrentar tomando en cuenta un análisis situacio- nal de su propia realidad, que involucre la determinación de potencialidades y amenazas. En este sentido sería ingenuo tratar de crear una lista de las acciones a seguir para construir este proceso.

Sin embargo, es factible delinear sucintamente los aspectos esenciales del proceso de desarrollo local a partir de su conceptualización - ya expuesta en este trabajo-y los elementos involucrados en el mismo.

En este sentido, puede establecerse en primer término como una condición básica para el logro del desarrollo local, la existencia de iniciativas locales; iniciativas que tienen que ver con la presencia de actores - agentes con intereses que aunque diversos tengan en común la búsqueda del aprovechamiento de las oportunidades de la localidad.

Estas iniciativas pueden conducir a la formación de "aglomerados sinérgícos" (Boisier, 1992: 114), compuestos porempresas, centros de investigación científica y tecnológica, gremios y corporaciones, con un alto grado de interconexión en sus intereses, estrategias de acción y repartición de resultados.

La posibilidad de que existan estos aglomerados a nivel local depende en gran medida de la capacidad que tenga el municipio de tomar sus propias decisiones, participar de ciertos recursos financieros, elaborar sus propias políticas públicas y establecer un marco legal adaptado a sus necesidades, es decir la existencia de un gobierno local dotado de autonomia relativa real. Esto sólo puede darse en el marco de la descentralización.

La descentralización política - territorial y administrativa es el medio a partir 
del cual se pueden crear espacios de interrelación entre Estado y sociedad, donde las modalidades de decisión permiten tener un mayor acercamiento a la situación objetivo de la decisión y una mayor posibilidad de negociación directa con los actores involucrados.

Un gobierno municipal autónomo podría actuar como un promotor para la configuración de los aglomerados sinérgicos, para dar paso a la construcción de un proyecto político local, fruto del consenso y la concertación de la comunidad. Este proyecto político debería enmarcarse en las metas de desarrollo de la comunidad nacional en la cual está inmerso el territorio, pero podría establecer objetivos ligados a necesidades regionales.

Los fines de desarrollo local podrían diseñarse tomando en cuenta la inclusión de requerimientos como:

- La creación de espacios políticos de participación.

- La construcción de la ciudadanía y la identidad local.

- La promoción de satisfacción de las necesidades básicas de la población y la equidad en la distribución de los recursos.

La promoción del crecimiento económico, productivo y competitivo de carácter sostenible y sustentable.

El primero de los requerimientos tiene que ver con la necesidad de democratización y la participación. La descentralización brinda la posibilidad de que las decisiones sean tomadas más cerca de los ciudadanos; pero no necesariamente que estos participen. Por esto es fundamental la generación de organizaciones y asociaciones corporativas que articulen representativa- mente las demandas de los individuos y que tengan acceso en la conformación e instrumentación del proyecto político local.

Es básico acotar que este entramado de organizaciones debe ser altamente legítimo, es decir que cuente con un alto grado de aceptación de los individuos y grupos sociales a los cuales representa, teniendo una estructura interna de tipo horizontal. De la misma manera, es condición indispensable que el grupo de excluidos sociales y culturales, por razones de ingreso o etnia, se involucren en este tipo de organizaciones.

Esto último está relacionado con la necesidad de creación de una verdadera ciudadania, que implique el reconocimiento de la diversidad cultural, es decir, el reconocimiento del otro como semejante, lo cual debería contribuir a la eliminación de cualquier tipo de discriminación: racial, sexual, etaria, socio-económica y política. Este reconocimiento tendría que operacionalizarse en la libre circulación de recursos de información, comunicación, socialización de poder y de producción de bienes y senvicios entre los miembros de la comunidad local.

El gobierno local autónomo tendría a la mano la pasibilidad de legislar y el aparataje administrativo para elaborar estrategias que permitan la consecución de estos requerimientos.

Una vía necesaria, es el rescate de la identidad colectiva local, que conlleve al referente histórico como generador de acumulación de capital cultural expresado en valores y tradiciones.

La base identitaria del desarrollo local no se limitará a recordar el pasado, sino recoger de este las experiencias para actuar sobre el presente y planificar el futuro.

Un tercer requerimiento del proceso en cuestión es como se mencionó, la pro- 
moción de la satisfacción de necesidades básicas de la población y la equidad en la distribución de los recursos. En este ámbito juega un papel fundamental la politica social local. Esta deberá procurar que todos los ciudadanos tengan igual acceso a condiciones minimas de bienestar. Estas acciones deberán estar en consonancia con las políticas públicas nacionales, que de manera sinérgica busquen la universalización de los servicios básicos (salud, educación, electricidad, agua potable, transporte público, entre otros) y la focalización en los casos que sea necesario intervenir para solventar las deficiencias de grupos vulnerables.

En la misma línea, el gobierno municipal y el "aglomerado" de actores-agentes del desarrollo local deberán iniciar una reforma educativa, que conduzca al encadenamiento entre el sistema educativo, el sistema de investigación cientifica y tecnológico y el sistema productivo, que produzca el establecimiento de metas interrelacionadas dirigidas a fomentar la productividad y competitividad no sólo de los sistemas mencionados, sino del recurso humano.

Lo anterior se inscribe a su vez en una política de empleo local que sea eficaz desde la escuela básica o primaria hasta la universidad, formando, capacitando, entrenando $y$ haciendo un monitoreo constante del capital humano que se prepara para ingresar al sector productivo.

Estos factores a su vez, se verán fortalecidos si se incluye una estrategia de crecimiento económico competitivo de carácter sostenible y sustentable. A nivel local esto tendrá como medio principal el apoyo al nacimiento y consolidación de microempresas y medianas empresas.

Se puede aprovechar el hecho, por ejemplo, de que muchas empresas grandes están asumiendo estrategias de "outsourcing", a través de las cuales se contratan los servicios de apoyo de otras compañías suscribiendo contratos a mediano y largo plazo con el fin de reducir los costos en que incurriría el contratante de realizar una actividad o proceso determinado, asi como mejorar la calidad e incrementar la productividad del mismo. Esto significaría utilizar el crecimiento, la experiencia y la creatividad de los pequeños y medianos empresarios que se han especializado en una función determinada a nivel manufacturero o de servicios. La contratación bajo outsourcing se diferencia de una relación tradicional cliente-proveedor al presentar las siguientes características según Rothery y Robertson $(1996,8)$ :

- Reuniones regulares de los miembros de la alta gerencia de ambas organizaciones.

- Los pagos dependen de los resultados del negocio o de los niveles de desempeño.

- Los contratos se establecen a cinco años o más.

- Revelación mutua de costos y beneficios.

- Participación en la planeación estratégica del otro.

- Dificultad para distinguir el personal de cada campaña.

- El socio no se selecciona con base en un proceso de un concurso competitivo. 
- Cada socio intenta formas para reducir costos totales.

- Cada socio agrega valor en forma real.

Asimismo, otro medio podria encontrarse en la introducción de modelos de calidad en las llamadas empresas informales, sobre todo las de tipo artesanal, complementándolos con ayuda financiera o gerencial por parte de agentes municipales, que conlleven a su transformación en pequeñas empresas formales con metas de expansión y crecimiento.

Aquí la capacitación de la mano de obra es fundamental, así como la generación de estudios a nivel de centros de investigación locales o nacionales que evalúen las condiciones actuales de estas empresas y propongan modelos de gestión adecuados a las mismas.

El desarrollo de las pequeñas y medianas empresas debería planificarse $e$ integrarse en la consecución de objetivos de largo plazo en cuanto a la creación de un sistema productivo local competitivo.

Los recursos necesarios para la inversión en estas metas tendrán que provenir de los actores - agentes del desarrollo local, que conforman la comunidad local, asi como de una estrategia nacional de desarrollo.

Por último, este conjunto de factores deberia producirse sin desestimar el impacto que sobre el ambiente y los recursos naturales tiene la instrumentación de una estrategia de transformación local como la que se expone. El control del uso que se hace del territorio, debe ser una de las características del proyecto político negociado por la comunidad local y la nacional.

Las vías más importantes serán la introducción de una legislación agresiva en materia ambiental y la revisión continua de su cumplimiento por parte del conjunto de individuos, grupos sociales y actores agentes que conforman la comunidad local. Parte de este "aglomerado sinérgico" tendrá que estar conformado no sólo por grupos de presión ecologistas sino por empresas privadas y/o públicas que se especialicen en servicios de mantenimiento y saneamiento ambiental.

\section{Amenazas y oportunidades del desarrollo local en América Latina}

Un proceso tan complejo como el descrito confronta en América Latina una serie de obstáculos y retos que deben ser superados en función de la consecución de metas de bienestar, equidad y democratización que exigen las actuales condiciones de las sociedades de este subcontinente.

Entre las amenazas más relevantes, es necesario señalar las relacionadas con factores estructurales contextualizados en los "estilos de desarrollo" prevalecientes en la región, caracterizados por la concentración y centralización de los recursos económicos, legales y políticos en las grandes capitales, no sólo las nacionales sino las estatales, provinciales o departamentales. Este estilo que han asumido los Estados Latinoamericanos ha conllevado a generar formas decisorias de tipo cupular ineficaces e inequitativas, en la medida que sobrecargan de información al aparataje burocrático centralizado dificultando una decisión adecuada a las especificidades de las subcolectividades regionales o locales; esto a su vez conlleva a la inequidad, puesto que a partir de estas decisiones alejadas de las 
realidades concretas se derivan efectos diferenciales que consolidan las desigualdades entre las comunidades que componen la comunidad nacional.

Esto exige una transformación que supere la descentralización espúrea y conlleve a una distribución efectiva del poder (democratización) del nivel nacional al regional y local y dentro de las localidades. La descentralización en este marco -como ya se ha planteado- tendría que asumir una modalidad en la que la participación comunitaria sea la columna vertebral en la formulación, ejecución y evaluación de las políticas públicas que operacionalizan la estrategia de desarrollo local, promocionándose un gobierno local autónomo e integrado por representantes de los diferentes grupos sociales. Como se verá más adelante, existen interesantes experiencias en Latinoamérica que pueden ser profundizadas y sistematizadas.

Es necesario acotar en segundo término, que el mantenimiento de estilos de desarrollo en condiciones de inequidad en América Latina se inserta en la posición que ha tenido esta zona geográfica en sus relaciones diplomáticas y comerciales con las sociedades industrializadas. La permanencia de esta posición dependiente es un factor que limita el potencial de las localidades de una comunidad nacional para generar iniciativas viables y expansivas que la sola aplicación de medidas de apertura indiscriminada al comercio mundial y la reducción del papel del Estado no pueden cambiar.

El cambio requerido a este nivel, implica que los procesos de transformación económica que se han iniciado en Latinoamérica en los últimos años, ven- gan acompañados de un consenso factible a nivel internacional sobre la importancia de coadyuvar a los procesos de desarrollo de estas comunidades nacionales, que trascienda por cierto las buenas intenciones y se convierta en una práctica de gobiernos e instituciones a nivel mundial.

Otra de las amenazas prevalecientes que pueden convertirse en una limitación para la consolidación de un proceso efectivo de desarrollo local en América Latina, está determinada por las actitudes culturales de las élites económicas, políticas, burocráticas $e$ intelectuales frente a lo que puede perfilarse como un redimensionamiento de las correlaciones $y$ líneas de poder $y$ autoridad en las comunidades nacionales y locales, asi como la emergencia de actores - agentes del desarrollo local con proyectos politicos integradores de iniciativas que trasciendan el interés elitesco para íncorporar objetivos de bienestar colectivo y estrategias de participación comunitaria.

En efecto, estas actitudes combinan prejuicios culturales muy arraigados, en parte subconscientes, valores inculcados a través de la educación, influencias ideológicas y normas internacionales dominantes, asi como la persecución de sus propios intereses (Wolfe, 1991: 24). Esta posición nutre una justificación tácita frente a condiciones de desigualdad extrema en la distribución de los recursos y a la exclusión de los menos privilegiados de una participación política efectiva.

Para superar esta condición es necesaria la comprensión por parte de estos importantes actores, de los beneficios que pueden producirse a nivel económi$c o$, político y social a través de un proceso 
de desarrollo local. Beneficios que presuponen la eficiencia y el crecimiento económi$c o$, la relegitimación o la construcción de legitimidad del sistema politico y la redefinición de la "conflictividad social", que podría pasar de ser una situación ligada a tensiones por la distribución de la riqueza, a una condición de negociación y diálogo en la búsqueda de soluciones a problemas colectivos.

En la medida en que las élites asuman estos requerimientos, su posición tendrá que orientarse a la cooperación, creando un panorama donde se establezcan intereses comunes, que impliquen una mayor cohesión social, configurándose también estos grupos sociales en actores - agentes del desarrollo local y nacional.

Si se reúnen las amenazas antes descritas y sus posibles soluciones, el desarrollo local se presenta distanciado de posibilidades objetivas de ser implantado. Sin embargo, existen algunas oportunidades generadas por la crisis de los años ochenta en el subcontinente Latinoamericano que podrían ser aprovechadas con el propósito de canalizarlas $e$ integrarlas en un proyecto local viable. Esto pasa por entender el desarrollo local no como una "situación" o un "estadio" de una sociedad, sino como un proceso, como una búsqueda constante y como un fin y un medio del progreso social de la comunidad nacional.

Es así como uno de los factores emergentes con la crisis de los últimos años en América Latina, ha sido el nacimiento de formas de organización de los sectores sociales menos privilegiados de la sociedad. Estos esfuerzos están ligados al deterioro constante de la calidad de vida; se han conformado no sólo para demandar del Estado mejoras en cuanto a la distribución de los recursos, sino como espacios donde pueden solventarse autónomamente necesidades de subsistencia.

Este tipo de organizaciones (cooperativas, asociaciones comunitarias de consumo y producción, asociaciones de vecinos) pueden convertirse en semillas de participación que promocionen la creación de una estrategia de desarrollo basada en estas iniciativas ( $y$ en otras que buscan satisfacer necesidades de autorrealización, como los grupos religiosos, deportivos, culturales y ecológicos). La sociedad civil tendría que pasar de ser la base o sustento pasivo de los actores políticos tradicionales, a un conjunto de iniciativas privadas que interactúan con otros actores tanto como demandantes o receptores de beneficios como promotores de sus propios satisfactores.

Otra de las oportunidades a ser tomadas en cuenta es la necesidad actual de legitimación o relegitimación de los partidos políticos. El deterioro progresivo de la aceptación de estas organizaciones se produjo por su incapacidad para cumplir con sus promesas electorales una vez que estaban en el poder. Sin embargo, los partidos políticos podrían cambiar el repudio generalizado frente a sus actividades, si redefinen su papel ante las nuevas formas de movilización comunitaria, siviendo como "negociadores" y no exclusivamente como "proveedores" en el proceso sistémico de generación y satisfacción de demandas.

En este ámbito, los partidos políticos deben constituirse en actores capaces de crear la viabilidad política necesaria para el proceso de desarrollo local, buscando el respaldo de los grupos relevantes del sistema político, movilizando este apoyo para comprometer los recursos políticos, admi- 
nistrativos, legales y financieros que sean necesarios. Asimismo, los partidos podrian servir como auspiciadores de la formulación del proyecto político local atendiendo a valores, percepciones, y objetivos de las partes interesadas y buscando la articulación de preferencias con los opositores.

Otra de las potencialidades a ser aprovechadas es la creciente formación de recursos humanos especializados en gerencia social, egresados de universidades u otros centros de investigación. Los "técnicos" se han capacitado para adecuar los medios o instrumentos de politica pública con los fines de la misma, identificando soluciones óptimas técnicamente para problemas concretos, tomando en cuenta los recursos disponibles, los factores ambientales que apoyan u obstaculizan la intervención y definiendo estrategias para su implantación; es decir construyen la "viabilidad técnica" de las políticas públicas.

Esta "racionalidad técnica" integrada con la viabilidad política, económica y la cultural es necesaria para proveer dinamismo y cubrir la multiplicidad de dimensiones del desarrollo local, pues de nada vale tener el interés para producir una estrategia como esta, si no se consideran los aspectos relacionados con la planificación, dirección y control que le den una orientación factible en la práctica.

Finalmente, un análisis de la situación actual que considere las oportunidades para generar un proceso de desarrollo local, tiene que hacer revisión de las primeras experiencias en cuanto a descentralización en los países Latinoamericanos. En los últimos años, se han puesto en práctica con el auspicio de gobiernos nacionales algunas modalidades de descentralización que aunque no poseen el carácter "sinérgico" como el aquí anotado, pueden ser tomados en cuenta como los primeros pasos que, de tener efectos profundos en las sociedades donde son implantados, pueden conducir al nacimiento de iniciativas locales autónomas.

El Centro de Documentación y Análisis de Información para el Desarrollo (CLAD) publicó recientemente (1997) un compendio de los principales logros de la descentralización en varios países. En este orden de ideas, se pueden revisar avances importantes, por ejemplo, en el caso de Honduras, país en el que a través de la Política y Programa Nacional de Descentralización y Fortalecimiento Municipal se ha propiciado el establecimiento y consolidación de instancias y mecanismos de participación ciudadana en la definición y manejo de asuntos y servicios públicos locales, tales como la iniciativa ciudadana de ordenanza municipal, juntas de usuarios de servicios públicos, entre otros. Se contempla además la introducción de "Mesas de Convergencia sobre el Desarrollo Municipal", que se perfilan como espacios permanentes de diálogo, concertación y negociación entre el gobierno nacional, los gobiernos municipales, el sector privado los gremios y la comunidad en general.

Asimismo, el proceso de descentralización en Bolivia ha adquirido características democratizadoras, por ejemplo, a partir de 1994 se aprobó y promulgó la "Ley de Participación Popular", como un instrumento que reconoce la personalidad juridica de Organizaciones Territoriales de Base (OTB) urbanas y rurales y la 
articulación de estas con los órganos públicos. Se definen como sujetos de estas formas de organización de participación popular a las comunidades campesinas, los pueblos indígenas y las juntas vecinales, todas ellas asociadas o agrupadas según sus usos, costumbres o disposiciones estatutarias. Entre los derechos de estas organizaciones se cuentan: proponer, pedir, controlar y supenvisar la realización de obras y la prestación de servicios públicos de acuerdo a las necesidades comunitarias; participar y promover acciones relacionadas a la gestión y preservación del medio ambiente, el equilibrio ecológico y el desarrollo sostenible; acceder a la información sobre los recursos destinados a la participación popular, entre otros.

Estas experiencias, entre las diversas presentadas por el CLAD, reflejan la existencia en el subcontinente de iniciativas que pueden servir como marco de referencia para formular una estrategia de desarrollo local en el marco de una estrategia de desarrollo social a nivel de comunidades nacionales.

Haciendo un balance entre amenazas y oportunidades es necesario mencionar que el saldo parece encaminarse a una tendencia positiva en la que las sociedades Latinoamericanas se verán en la obligación de propiciar cambios en sus estilos de desarrollo para superar las brechas existentes a nivel intemo y las existentes entre las comunidades nacionales de esta parte del mundo y los países industrializados.

\section{Conclusiones}

La discusión sobre desarrollo local en la actualidad lleva más a la construcción de preguntas que de respuestas.
El desarrollo local, como se ha visto, es un proceso multidimensional, si se le quiere observar desde una perspectiva compleja y sistémica como la anotada en este trabajo, pero su viabilidad será inexistente si no se combinan los elementos iniciales ya identificados: la iniciativa local autónoma y la descentralización. El segundo de estos aspectos tendrá que ser una decisión también de la comunidad nacional que tenga como finalidad la búsqueda de un crecimiento de la sociedad en conjunto y no de sectores específicos, cubriendo las dimensiones sociales, políticas, culturales y no sólo la económica.

En este ámbito resulta básica la superación de los obstáculos presentes en los ámbitos internos y externos a la sociedad que inicia el proceso en cuestión y el aprovechamiento de las potencialidades con las que cuente.

La integración de estos factores puede conllevar a la configuración de un proyecto político local en el que se establezca como meta la búsqueda de una vida mejor para todos los ciudadanos de la comunidad local, en armonia con su ambiente y en total correspondencia con los intereses de la nación a la cual "pertenecen".

\section{Referencias bibliográficas}

Arocena, José (1989). "Descentralización e Iniciativa, una Discusión Necesaria", Cuadernos del CLAEH, No. 51, pp. 43-56, Centro Latinoamericano de Economia Humana (CLAEH), Montevideo - Uruguay .

Arocena, Jose (1995). "EI Desarrollo Local: Un Desafió Contemporáneo". Centro Latinoamericano de Economía Humana (CLAEH), Universidad Católica del Uru- 
guay, Editorial Nueva Sociedad, Caracas - Venezuela.

Boisier, Sergio (1992). "Las Relaciones entre Descentralización y Equidad", Revista de la CEPAL, No. 46, pp. 113-131, Comisión Económica para América Latina y el Caribe, Naciones Unidas, Santiago de Chile.

Calderón, Fernando; Hopenhayn, Martin y Ottone, Ernesto. (1994). "Una Perspectiva Cultural de las Propuestas de CEPAL", Revista de la CEPAL, No. 52, pp. 191 - 205, Comisión Económica para América Latina y el Caribe, Naciones Unidas, Santiago de Chile.

Cárdenas, Nersa (1996). "El Desarrollo Local: Su Conceptualización y Procesos", Fundación Escuela de Gerencia So- cial. Mimeografiado, Caracas - Venezuela.

Centro Latinoamericano de Administración para el Desarrollo. (1997). "La Reforma del Estado Abordada Globalmente y a Través de la Descentralización Político - Administrativa". CLAD, Caracas - Venezuela.

Rothery Brian y Robertson lan (1996). "Outsourcing". Limusa - Noriega Editores. México.

Touraine, Alain (1992). "Crítica de la Modernidad". Fondo de Cultura Económica, México.

Wolfe, Marshall. (1995). "Perspectivas sobre la Equidad", Revista de la CEPAL, No. 44, pp. 21 - 37, Comisión Económica para América Latina y el Caribe, Naciones Unidas, Santiago de Chile. 